International Journal of Statistics and Analysis.

ISSN 2248-9959 Volume 11, Number 1 (2021), pp. 1-4

(C) Research India Publications

https://dx.doi.org/10.37622/IJSA/11.1.2021.1-4

\title{
Generalized Synthetic Estimator under Simple Random Sampling
}

\author{
Dr. Sangeeta Malik \\ Dept. of Mathematics BMU, Rohtak, Haryana, India.
}

\begin{abstract}
A synthetic estimator studied by Gonzalez (1973) is based on an assumption that small domains under consideration are similar in characteristics to some other domain, usually a larger domain which contains it. A synthetic estimator may be based on the auxiliary data besides the population size of the small domain. In this paper, the researcher has considered the problems of population estimation by using small area technique and auxiliary information. The researcher proposed quadratic product estimator and generalized ratio cum product estimator under simple random sample scheme. The justification of this proposed estimator is more efficient than usual estimators under certain condition has been given with empirical study.
\end{abstract}

Keywords: synthetic estimator, quadratic product estimator, ratio cum product estimator etc.

\section{INTRODUCTION}

For any sample design, Let a finite population $U=(1, \ldots . . . . . N)$ is divided into ' $\mathrm{A}$ ' non-overlapping domains $U_{a}$ of size $N_{a}(\mathrm{a}=1, \ldots ., \mathrm{A})$. A simple random sample without replacement (SRSWOR) $S=(1, \ldots \ldots i, \ldots, n)$ is divided into 'A' non overlapping domains $S_{a}$ of size $n_{a}(a=1, \ldots . ., A)$;

$$
\therefore \sum_{a=1}^{A} N_{a}=N \text { and } \sum_{a=1}^{A} n_{a}=n
$$

Here, $\bar{Y}$ and $\bar{X}$ are population mean for the main and the auxiliary variables. $\bar{Y}_{a}$ and $\bar{X}_{a}$ are the population mean for the main and the auxiliary variables for the small area ' $a$ '. 


\section{PROPOSED ESTIMATORS IN SIMPLE RANDOM SAMPLING}

I.I First, we propose the quadratic product type synthetic estimator

$$
\bar{y}_{s y n, q p, a}=\bar{y}\left(\frac{\bar{x}}{\bar{x}_{a}}\right)^{2}
$$

Let $\quad e_{0}=\frac{\bar{y}-\bar{Y}}{\bar{Y}} \quad, \quad e_{1}=\frac{\bar{x}-\bar{X}}{\bar{X}}$ and $E\left(e_{0}\right)=0=E\left(e_{1}\right)$

The above estimator can be biased unless the following synthetic assumption is satisfied

$$
\left\{\begin{array}{l}
\bar{Y}_{a} \doteq \bar{Y} \\
\bar{X}_{a} \doteq \overline{\mathrm{X}}
\end{array}\right.
$$

$\bar{y}_{s y n, q p, a}=\bar{Y}\left(1+e_{0}\right)\left(\frac{\bar{X}\left(1+e_{1}\right)}{\bar{X}_{a}}\right)^{2}$

$\operatorname{Bias}\left(\bar{y}_{s y n, q p, a}\right)=E\left[\bar{y}_{s y n, q p, a}\right]-\bar{Y}_{a}$

$\operatorname{Bias}\left(\bar{y}_{s y n, q p, a}\right)=\bar{Y}_{a}\left(\frac{1-f}{n}\right)\left(C_{x}^{2}+2 C_{x y}\right)$

M.S.E. $\left(\bar{y}_{s y n, q r, a}\right)=\mathrm{E}\left[\bar{y}_{s y n, q r, a}-\bar{Y}_{a}\right]^{2}$

If the synthetic assumption (2.3) is satisfied, then the above expression reduces to

$$
\text { M.S.E. }\left(\bar{y}_{s y n, q p, a}\right)=\bar{Y}_{a}^{2}\left(\frac{1-f}{n}\right)\left(C_{y}^{2}+4 C_{x}^{2}+4 C_{x y}\right)
$$

\section{Proposed generalized ratio cum product synthetic estimator}

Here, we propose generalized ratio cum product synthetic estimator using auxiliary variable

$$
\bar{y}_{s y n, r p, a}=\bar{y}\left[\left(\frac{\bar{x}}{\bar{x}_{a}}\right)^{1+\beta}+\left(\frac{\bar{x}}{\bar{x}_{a}}\right)^{\beta-1}\right] / 2 \quad ; \quad-1<\beta<1
$$

Case (i) when $\quad \beta=-1$, the above estimator (5) becomes

$$
\bar{y}_{\text {syn,rp,a}}=\bar{y}\left[1+\left(\frac{\bar{x}_{a}}{\bar{x}}\right)^{2}\right] / 2
$$

Case (ii) when $\quad \beta=0$, the above estimator (5) becomes

$$
\bar{y}_{\text {syn }, r p, a}=\bar{y}\left[\left(\frac{\bar{x}}{\bar{x}_{a}}\right)+\left(\frac{\bar{x}_{a}}{\bar{x}}\right)\right] / 2
$$

Case (iii) when $\quad \beta=1$, the above estimator (5) becomes

$$
\bar{y}_{\text {syn }, r p, a}=\bar{y}\left[\left(\frac{\bar{x}}{\bar{x}_{a}}\right)^{2}+1\right] / 2
$$


Now, substituting the expression in terms of $\mathrm{e}^{\mathrm{s}}$ up to first degree approximation, we get

$$
\bar{y}_{s y n, r p, a}=\bar{Y}\left(1+e_{0}\right)\left[\left(\frac{\bar{X}\left(1+e_{1}\right)}{\bar{X}_{a}}\right)^{1+\beta}+\left(\frac{\bar{X}\left(1+e_{1}\right)}{\bar{X}_{a}}\right)^{\beta-1}\right] / 2
$$

$\operatorname{Bias}\left(\bar{y}_{s y n, r p, a}\right)=\frac{\bar{Y}_{a}}{2}\left(\frac{1-f}{n}\right)\left[\left(\beta^{2}-\beta+1\right) C_{x}^{2}+2 \beta C_{x y}\right]$

If the synthetic assumption (3) is satisfied, then the above expression reduces to

$$
\text { M.S.E. }\left(\bar{y}_{s y n, r p, a}\right)=\bar{Y}_{a}^{2}\left(\frac{1-f}{n}\right)\left(C_{y}^{2}+\beta^{2} C_{x}^{2}+2 \beta C_{x y}\right)
$$

\section{Optimum Estimator}

The optimum estimator of generalized ratio cum product synthetic estimator using simple random sampling scheme is up to first degree approximation after satisfied the synthetic assumption (3), for which M.S.E $\left(\bar{y}_{s y n, r p, a}\right)$ is minimized and so minimize the expression (6) w.r.t. ' $\beta$ ' gives optimum value

$$
\beta=-\rho \frac{C_{y}}{C_{x}}
$$

So put the value of ' $\beta$ ' in equation (6), then the minimum variance is as

$$
\text { M.S.E. }\left(\bar{y}_{s y n, r p, a}\right)_{o p t}=\bar{Y}_{a}^{2}\left(\frac{1-f}{n}\right) C_{y}^{2}\left(1-\rho^{2}\right)
$$

Here, $C_{x y}=\rho C_{x} C_{y}$

\section{EMPIRICAL STUDY}

To study the efficiency of the proposed estimators we consider this data for small area statistic given in Gujrati, D.N. (2004, pp. 433)

\section{POPULATION}

$y:$ Average miles per gallon

$x$ : Cubic feet of cab space

The required population parameters are:

$\bar{Y}_{a}=112.4568, \quad C_{y}=.1248, \rho_{x y}=.967, \mathrm{~N}=81$

$\bar{X}_{a}=117.4691, C_{x}=.4831, n^{\prime}=21, \mathrm{n}=9$

The relative efficiency of the proposed estimators is calculated and compare with respect to the usual simple (mean per unit), ratio and product synthetic estimators as 
shown in table.

TABLE

\begin{tabular}{|c|c|c|c|c|}
\hline \multirow{2}{*}{$\begin{array}{c}\text { Proposed } \\
\text { Estimator }\end{array}$} & $\beta$ & \multicolumn{3}{|c|}{ Population } \\
\cline { 2 - 5 } & & Mean & Ratio & Product \\
\hline $\bar{y}_{\text {syn,qp,a}}$ & - & 183.102 & 322.811 & 108.832 \\
\hline $\bar{y}_{\text {syn,rp,a }}$ & .67 & 144.954 & 255.554 & 86.157 \\
\hline $\bar{y}_{\text {syn, } r p, a}$ & .9 & 161.588 & 284.881 & 96.044 \\
\hline $\bar{y}_{\text {syn, } r p, a}$ & .2 & 112.324 & 198.028 & 66.763 \\
\hline
\end{tabular}

\section{CONCLUSION}

The researcher after going through this population, it is proposed that generalized ratio cum product synthetic estimators in simple random sampling is better than simple (mean per unit) and ratio synthetic estimators but not with product synthetic estimator because the choice of constant variable and correlation coefficient. In case when $\beta=.5$ and $\beta=-.5$ then generalized ratio cum product synthetic estimators are equally efficient. But when $\beta=0$, then the proposed generalized synthetic estimators are equally efficient to simple synthetic estimator. Using this population the quadratic product (SRS) synthetic estimator has highest relative efficiency with respect to the simple, ratio and product synthetic estimator.

\section{REFERENCES}

[1] Cochran, W.G. (1977). Sampling Techniques. 3rd edn., John Wiley: New York.

[2] Ghangurde, P.D. and Singh, M.P. (1977). Synthetic Estimators in Periodic Household Surveys, Survey Methodology 3,(151-181).

[3] Gonzalez, M.E and Waksberg, J. (1973), Estimation of the error of Synthetic Estimates, Paper Presented at the first meeting of the International association of Survey Statisticians at Vienna, Austria.

[4] Gujarati, D.N. (2004). Basic Econometrics, The McGraw-Hill Companies.

[5] Rao, J.N.K. (2003) Small Area Estimation. Wiley, New York.

[6] Ray S.K. and Sahai Ajit (1978). On Use of Auxiliary Information in Sampling, Jour. of Stat Res. Vol. 12 Nos. 1and 2

[7] Tikkiwal, G.C. and Ghiya, A. (2000). A Generalized class of synthetic estimators with application to crop acreage estimation for small domains, Biometrical Journal, 42 (7), 865-876. 